\title{
Spécificité de l'odontologie hospitalière en milieu carcéral
}

\section{Specificity of hospital dentistry in prison}

\section{MOTS-CLEFS :}

- Soins dentaires, prison, hôpital public, éducation thérapeutique, détenus, toxicomanie.

\section{KEYWORDS:}

- Dental care, prison, public hospital, patient education, prisoners, drug addiction.

AOS 2013;263:4-7

DOI: $10.1051 / \mathrm{aos} / 2013302$

(C) EDP Sciences 2013

\section{Résumé}

La prise en charge des soins dentaires en milieu carcéral est effectuée par le centre hospitalier le plus proche.

Après un bilan bucco dentaire d'accueil au moment de l'incarcération, le détenu peut demander ò tout moment à se faire soigner.

Si les soins en prison restent les mêmes que ceux pratiqués à l'extérieur, il existe cependant un certain nombre de spécificités propres au milieu carcéral.

La nécessité de soins dentaires est aggravée par l'indigence, la précarité ou l'usage de produits stupéfiants.

Parfois des actes de violence entre détenus génèrent des plaies ou des fractures.

Le vieillissement de la population carcérale s'observe également avec ses pathologies associées.

Enfin, selon les établissements de peine, les conditions sont plus ou moins propices à l'initiation ou ò la perpétuation de plans de traitement sophistiqués.

Quoiqu'il en soit, il est indispensable de pouvoir soigner tout détenu comme n'importe quel autre patient. Il ne doit exister aucun obstacle à un soin de qualité.

C'est la contribution des soignants pour que le détenu puisse entreprendre un travail de réinsertion et espérer éviter la récidive.

Si la prison est un lieu de privation de liberté, ce ne doit pas être un endroit de privation de dignité.

\section{Abstract}

The dental treatments in prison are made by the closest hospital center. After a dental balance sheet at the entrance, the prisoner can ask at any time to be looked after.

If the care in prison stay the same that those practised outside, it exists however a lot of specificities in prison environment.

The necessity of dental treatment is deteriorate by the beggary, the precariousness or drug addiction. Sometimes acts of violence between prisoners generate wounds or fractures. The ageing of the prison population also goes with its associated pathologies.

Finally, according to the establishments of punishment, the conditions are more or less convenient to the initiation or perpetuation of sophisticated treatment plans.

Nevertheless, it is essential to treat all prisoners as well as other patients. There should be no barriers to quality care. This is the carer's contribution to enable the prisoners to undertake rehabilitation work and hope to avoid recidivism.

If the prison is a place of loss of liberty, it should not be a place of deprivation of dignity.

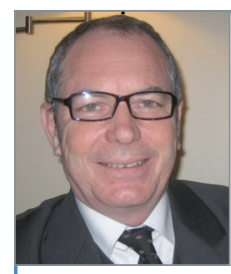

- Dominique Orphelin,

Praticien hospitalier, Spécialiste Qualifié en Médecine Bucco Dentaire UCSA-D2

7 avenue des Peupliers

91705 Sainte-Geneviève-des-Bois Cedex 


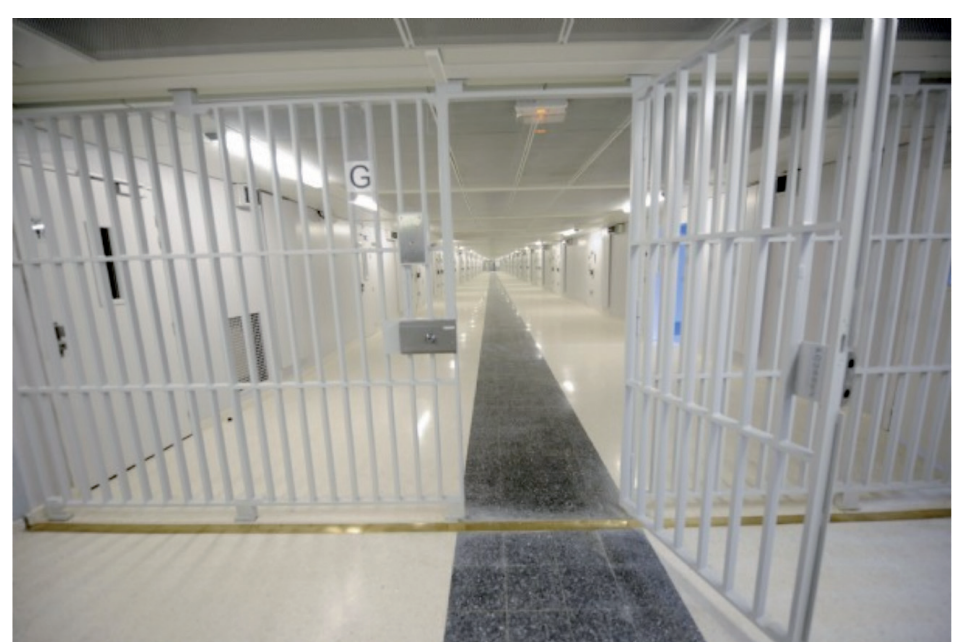

$\triangle$ Fig. 1 :

La prison de Fleury-Mérogis.

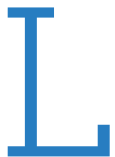

a prise en charge des personnes placées sous main de justice ne diffère pas de celle de n'importe quelle personne nécessitant des soins. Depuis la loi $n^{\circ}$ 94-43 du 18 janvier 1994, l'hôpital public prend en charge le suivi médical des personnes détenues. Des moyens matériels et humains ont été développés au sein d'Unités de consultations et de soins ambulatoires (UCSA) à l'intérieur des établissements pénitentiaires afin de s'occuper au plan sanitaire de la population carcérale. Les soins courants y sont prodigués. Certaines spécialités médicales sont parfois présentes. Les autres consultations plus spécifiques sont programmées vers d'autres hôpitaux en cas de nécessité. Parallèlement, il existe un service médico-psychiatrique.

\section{LA PRISE EN CHARGE DENTAIRE}

Des cabinets dentaires ont été installés ou rénovés dans la plupart des établissements pénitentiaires. Des installations fonctionnelles munies d'appareils de radiographie rétro-alvéolaire permettent une offre de soins courante. Les chirurgiens-dentistes, praticiens hospitaliers ou attachés, apportent leur savoir-faire et leur dévouement afin que la prise en charge soit la plus complète possible. Il n'y a pas d'assistante dentaire car cette profession n'est pas inscrite au Code de la santé publique.

\section{L'ACCÈS AUX SOINS}

Chaque arrivant dans un établissement pénitentiaire bénéficie d'une visite médicale d'entrée. La nécessité de soins dentaires est décelée dès l'arrivée en prison grâce à cette visite d'accueil.

Ce bilan devrait être fait par un chirurgien-dentiste afin d'apprécier les besoins éventuels exacts, de planifier les consultations et de proposer un suivi personnalisé. Malheureusement, les effectifs des praticiens étant trop faibles, cette visite est le plus souvent déléguée au personnel infirmier qui se contente de seulement demander si le détenu a simplement mal aux dents ou s'il éprouve le besoin de soins dentaires.

Larrivée en prison s'accompagne souvent d'un stress lié au choc de la détention après la période de garde-à-vue et la présentation au juge.

La préoccupation principale d'un arrivant est son «installation» : aura-t-il la télévision, des cigarettes, son traitement pour dormir ou ses médicaments de substitution?

Cette période ne constitue pas toujours un climat propice à la proposition d'un bilan bucco-dentaire. Le détenu lui-même pourra solliciter par la suite une consultation dentaire en adressant au service médical une demande écrite (imprimé pré-rempli avec des dessins pour une compréhension universelle).

Le délai d'obtention d'un rendez-vous est variable selon les établissements. Cette attente est comparable à celle de l'extérieur (elle est parfois même plus courte).

Mais ce délai peut être modulé en fonction du comportement du détenu : une personne agitée ou entretenant des rapports de force avec les surveillants ou qui serait impolie ou insultante (à l'encontre du personnel pénitentiaire ou du personnel médical) pourra voir son accès à l'unité de soins perturbé ou retardé.

Cependant, en cas d'urgence, le détenu est dirigé aussitôt vers le service médical.

La demande écrite est importante car elle correspond à une démarche volontaire de la part du patient dans une population très peu habituée à se faire suivre sur le plan sanitaire.

\section{SPÉCIFICITÉ DE LA DEMANDE DE SOINS EN PRISON}

La nécessité de soins dentaires est très importante en prison. En 2000, à la prison de Loos, $87 \%$ des détenus avaient besoin de soins dentaires. L'état de la bouche était considéré comme mauvais ou très mauvais dans $50 \%$ des cas, moyen dans $32 \%$ des cas et bon dans seulement $28 \%$ des cas [1].

Le suivi dentaire à l'extérieur dépend souvent du niveau social du détenu. Les personnes intégrées normalement dans la société, ayant un travail, une famille, se font habituellement suivre régulièrement pour leur santé en général et les soins dentaires en particulier.

Hélas, chez la grande majorité des détenus, on constate beaucoup de précarité, des toxicomanies diverses et une grande prévalence des maladies transmissibles à virus hématogènes. Ces personnes ont rarement eu d'éducation à la santé et ont une grande méconnaissance de la réalité des soins.

Ayant une vie sociale extérieure différente, vivant en marge de la société, neetant pas intégrés dans les structures sociales conventionnelles, ils ont parfois une approche des soins un peu particulière : ils se font soigner uniquement quand ils ont mal, ils sont adeptes de réseaux de soins parallèles et clandestins ou bien ils se dirigent vers des praticiens à l'étranger pour des raisons financières le plus souvent (mais aussi pour 
des questions de confiance ou à cause de la barrière de la langue).

Autre paradoxe, bien que la demande de soins soit effective, ils vont renoncer pour des raisons qui ne sont pas toujours très claires : la peur, les légendes carcérales et les bruits de couloir (les praticiens ne sont pas diplômés) ou l'espoir de sortir rapidement et de se faire soigner dehors (alors qu'ils n'ont aucun suivi extérieur régulier).

Souvent ils préfèrent idéaliser et remettre à plus tard : "quand je sortirai, je me ferai tout refaire!"

Pourtant leur apparence extérieure les préoccupe : beaucoup de détenus réclament des blanchiments de leurs dents.

\section{SPÉCIFICITÉ LIÉE AUX PATIENTS}

\section{Les toxicomanes}

La toxicomanie qui est fréquente et parfois considérable n'est pas propre à la prison mais liée à l'origine des détenus.

Ce n'est pas une population homogène. Se trouvent souvent intriqués toxicomanie, sida, misère économique et sociale, analphabétisme et désordres comportementaux.

Un détenu sur trois déclare consommer régulièrement de la drogue ( $15 \%$ en consomment plusieurs), souvent sur fond de tabagisme ( $80 \%$ sont fumeurs). Un détenu sur trois déclare une consommation régulière d'alcool. Leur état bucco-dentaire est souvent délabré, presque toujours négligé ( $80 \%$ ont besoin de soins et $100 \%$ chez les toxicomanes aux opiacés) [4].

Leur plongée dans la drogue les fait échapper aux réalités quotidiennes, le reste du temps étant consacré à la recherche de stupéfiants ou à la recherche d'argent pour l'obtenir (vols, trafics divers).

\section{Les mineurs}

S'ils ont pu avoir un suivi plus ou moins régulier durant leur enfance, ce suivi s'est détérioré lorsqu'ils ont échappé à la tutelle parentale (parfois précocement). C'est à partir de ce moment que leur bouche a pu se dégrader rapidement : soins non poursuivis, traitements d'orthodontie stoppés ou non entrepris.

Le bilan bucco-dentaire systématique des arrivants mineurs à la maison d'arrêt de Fleury-Mérogis au premier trimestre 2012 a montré un indice CAO de 2,55 (à comparer à l'indice $\mathrm{CAO}$ des détenus non toxicomanes qui est de 9,28 et à celui des héroïnomanes qui est de 12,98 à la prison de Loos) [2].

D'autre part, les jeunes vivent souvent dans un climat de déchaînement et de violence extrêmes (loi du plus fort, règlements de compte divers) où des bagarres plutôt musclées ont des conséquences traumatiques dentaires ou maxillo-faciales sévères sans qu'il y ait toujours un traitement médical ou chirurgical (refus de se signaler, refus de soins).

\section{Les personnes âgées}

Alors que la population carcérale en France est plutôt jeune (lâge médian des hommes est de 32 ans, celui des femmes est de 35 ans), on remarque depuis quelques années un vieillissement des personnes incarcérées (7800 détenus ont plus de 50 ans dont 2400 ont plus de 60 ans sur une population recensée de 67700 détenus en avril 2010) [3]. Cette population vieillissante amène un cortège de pathologies dentaires associées qu'il faut pouvoir prendre en charge.

\section{Les personnes étrangères}

Un certain nombre de détenus, d’origine étrangère, ne parlent absolument pas le français ni d'autres langues européennes. Leur prise en charge sanitaire est malaisée car la communication avec eux est difficile voire impossible.

Parfois un de leur codétenu peut servir d'interprète mais alors le secret médical ne peut être respecté.

\section{Les établissements pénitentiaires}

Il existe plusieurs types d'établissements pénitentiaires que l'on peut classer essentiellement en deux catégorie: les maisons d'arrêt et les établissements de peine

\section{Les maisons d'arrêt}

On y trouve les personnes "prévenues» (en mandat de dépôt pendant l'instruction de leur affaire, avant leur jugement). On y rencontre également des gens condamnés à de courtes peines ou en cours de transfert d'une prison à une autre. Certains détenus en fin de peine peuvent se retrouver en maison d'arrêt.

Cette population n'est pas censée séjourner longtemps dans ce type d'établissements (6 à 9 mois en moyenne). Le profil du détenu ne permet pas de toujours savoir quelle sera la durée exacte de son incarcération.

Dans ces conditions, il est assez délicat d'entreprendre des plans de traitement sophistiqués.

Le soin dentaire y relève souvent du soin de première nécessité.

\section{Les établissements de peine}

En revanche, dans ces établissements (centres de détention, centrales), le détenu a été jugé. Il ne se trouve plus dans l'incertitude du mandat de dépôt. Il connaît la durée exacte de sa peine et devient, sinon serein, du moins fixé.

Il peut alors entreprendre un travail de reconstruction et de réinsertion.

La remise en état de sa bouche passe alors un nouveau cap où le patient devient plus réceptif et donc plus motivé. Il devient aussi plus respectueux des rendez-vous.

\section{Autres spécificités}

- Les traitements psychotropes, fréquemment prescrits, qui induisent une plus ou moins grande hyposialie. 
- La consommation boulimique de sucreries : les confiseries apportent aux détenus de la douceur et du bien-être.

- Les détenus sous traitement de substitution aux opiacés au moyen du chlorhydrate de méthadone dont la forme galénique en sirop est très riche en saccharose.

\section{RÉFLEXION}

Il est vrai que milieu libre et milieu fermé ont toujours été opposés : notre société n'a que peu d'informations sur la réalité de la vie à l'intérieur de la prison et ne cherche (peut-être) pas à s'en procurer, jugeant que le monde carcéral est souvent irrécupérable. Cet isolement ne permet pas de reconnaître la qualité du travail effectué par les différentes catégories de personnels. Il y a ainsi de nombreux préjugés concernant les détenus, ce qui rend difficile leur réinsertion et facilite la récidive. C'est une population défavorisée, souvent toxicomane, dont il s'avère nécessaire d'étudier les raisons de leur incurie bucco-dentaire avant l'incarcération. Ceci en dépit d'un dispositif médico-social dense à l'extérieur : les difficultés que nous rencontrons en milieu pénitentiaire concernent moins la prison elle-même que la société civile dans son ensemble.

Cependant, le système pénitentiaire ne doit pas aggraver les souffrances inhérentes à la situation de privation de liberté [5]. Aussi est-il indispensable de voir le patient avant de voir le détenu.

La prise en charge collégiale pluridisciplinaire du patient au sein des unités de soins est une approche très intéressante qui permet un accompagnement thérapeutique personnalisé. Cette démarche permet de ne pas penser la prison comme seulement un lieu de privation de liberté mais aussi comme une structure qui va réinsérer les personnes dans le circuit social.

Lamélioration technique des soins dentaires est cô̂teuse. Ne pas faire profiter les détenus des progrès de la science est inacceptable quand on considère que tout patient a droit à la santé et qu'il faut réduire les inégalités d'accès aux soins.

Cependant il faut rester réaliste et honnête face aux coûts des techniques novatrices en chirurgie dentaire. Nous nous souvenons tous du précepte "primum non nocere», mais ne rien faire, n'est-ce pas nuire aussi ?

Les contraintes financières ne doivent pas nous conduire au renoncement et par conséquent à encore plus d'exclusion de celui qui est déjà au ban de la société. Afin d'éviter d'accentuer sa marginalisation, nous devons accompagner notre patient-détenu et faire en sorte qu'il adhère à notre plan de traitement. L'information, l'explication, léducation à la santé sont d'une importance primordiale et doivent être cultivées et promues au même titre que le soin lui-même. Cet accompagnement qui se fait avec l'accord et la participation du patient lui permettra de retrouver une estime de soi ainsi qu'un statut social. Sinon la prise en charge du patient se soldera par un échec.

La personne emprisonnée est un citoyen qui doit

bénéficier des mêmes possibilités d'accès aux soins qu'une personne libre [3]. Pourtant les contraintes financières nous rappellent bien souvent que le détenu reste une "charge» plutôt qu'une "attention». Le soignant doit cependant rester attaché aux exigences et aux valeurs du soin en dépit des divers obstacles ou autres pressions. Il doit se consacrer avec générosité et compétence, même s'il est parfois tenté de renoncer là où nos tutelles abdiquent. Tout ce qui peut affecter la qualité de nos soins est inacceptable et injustifiable. Il est indécent de nous en invoquer le coût. Nous devons résister à toute considération qui risque de dévaluer la qualité du soin.

Méditer sur le sens du soin (dentaire) en prison, c'est réfléchir sur le véritable engagement social et politique de notre société vis-à-vis de la population carcérale.

\section{CONCLUSION}

Une prison est un lieu de privation de liberté mais pas un endroit de privation de dignité.

Au-delà du soin (réponse ciblée à un besoin précis), il est indispensable d'avoir une écoute bienveillante, d'entamer un dialogue constructif et de mettre en place une prise en charge globale.

Il faut savoir donner priorité au patient, le soulager, le respecter sans discrimination aucune et sans imposer ses opinions.

Si la prison n'est pas un lieu dédié au soin, elle reste cependant un endroit où l'on peut initier une démarche thérapeutique, la perpétuer et de ce fait éduquer une population. Car soigner seulement «l'urgence» manque cruellement d'ambition.

Bibliographie

[1] Becart-Robert A, Cogo M, Tourmel G, Moutel G, Hedouin V, Gosset $\mathrm{D}$, Activité dentaire à l'UCSA de Loos. Bilan de 9 années d'exercice. Communication : $4^{e}$ Congrès des UCSA de l"Est, Nancy, octobre 2000.

[2] Becart A, Hedouin V, Martin-Bouyer L, Revuelta E, Gosset D, Oral state of health in drug addicts : a carceral survey in Lille, France. J Forens Odont-Stomat, 19971997.

[3] Obrecht $O$, Des progrès pour la santé en prison. Ceras Revue Projet $\mathrm{n}^{\circ}$ 269, juin 2002.

[4] Questions de l'Assemblée Nationale, $n^{\circ} 82088$ publié au JO du 22 juin 2010, page 6865, réponse publiée au JO du 21 septembre 2010, page 10402.

[5] Rapport de la Commission d'enquête du Sénat. Les conditions de détention dans les établissements pénitentiaires en France, juin 2000.

[6] Règles pénitentiaires européennes, Conseil de l'Europe, 1987. 